\title{
System Design of an Insertable Robotic Effector Platform for Single Port Access (SPA) Surgery
}

\author{
Kai Xu, Roger E. Goldman, Jienan Ding, Peter K. Allen, Dennis L. Fowler and Nabil Simaan
}

\begin{abstract}
This paper presents a novel design and preliminary kinematic analysis of an Insertable Robotic Effector Platform (IREP) for Single Port Access (SPA) Surgery. The IREP robot can be deployed into body cavity through a $\varnothing 15 \mathrm{~mm}$ skin incision to perform SPA procedures. It consists of two snake-like continuum robots as slave surgical assistants for tissue manipulation, two parallelogram mechanisms for the continuum robots' placement, and one controllable stereo vision module with integrated light source for depth perception and tool tracking. Design considerations and alternatives, calculations and preliminary simulations of this 17-DoF surgical robotic system are presented in this paper. The overall control system hierarchy for tele-manipulation using the IREP robot is also presented.
\end{abstract}

\section{INTRODUCTION}

$\mathrm{L}$ APAROSCOPIC and other minimally invasive surgeries have successfully reduced patients' post operative pain, complications, hospitalization time and improved cosmesis $[1,2]$. During most laparoscopic procedures, two or more incisions are used for surgical instruments, visualization, and insufflation [3]. Before Natural Orifice Transluminal Endoscopic Surgery (N.O.T.E.S) which eliminates all the skin incisions can be widely applied to broader procedures and population [4-6], researchers and surgeons may focus on Single Port Access (SPA) surgeries which reduce the number of skin incisions to one and therefore may improve surgical outcome compared manual laparoscopic procedures $[7,8]$.

Most existing robotic surgical systems are designed for minimally invasive laparoscopic procedures $[9,10]$. Although robotic assistance has greatly enhanced surgeons' capabilities in performing standardized laparoscopic techniques [11], these existing robotic systems are not suitable for SPA surgeries due to the large size of their instruments and lack of overarching control and collision avoidance among their multiple arms. Therefore, manual

Manuscript received Mar $9^{\text {th }}, 2009$. This work is supported by NIH grant \#R21 EB007779.

Kai Xu (Ph.D Candidate, kx2102@columbia.edu), Jienan Ding (Ph.D, dj2253@columbia.edu), and Nabil Simaan (corresponding author) (Ph.D., ns2236@,columbia.edu) are with the Department of Mechanical Engineering, Columbia University, New York, NY 10027, USA.

Roger E. Goldman (MD/Ph.D Candidate, reg2117@columbia.edu) is with Department of Biomedical Engineering and College of Physicians \& Surgeons, Columbia University, New York, NY 10027, USA

Peter K. Allen (Ph.D, allen@cs.columbia.edu) is with Department of Computer Science, Columbia University, New York, NY 10027, USA

Dennis L. Fowler (M.D, dlf91@,columbia.edu) is with Department of Surgery, Columbia University New York, NY 10032, USA.
SPA surgeries are currently limited to just a few academic centers using specifically modified manual laparoscopic instruments (such as RealHand ${ }^{\mathrm{TM}}$ ) $[8,12,13]$.

To meet the challenges of SPA surgeries, an Insertable Robotic Effector Platform (IREP) (Fig. 1) is designed by merging our enabling technologies of endoscopic imaging [14-17] and distal dexterity enhancement [18-22]. The IREP robot is a completely new design which integrates stereo vision and dexterous end effectors for surgical interventions. It consists of two five-DoF snake-like continuum robots, two two-DoF parallelogram mechanisms, and one three-DoF stereo vision module. The system will decrease the complexity of abdominal SPA procedures, such as cholecystectomy, appendectomy, etc.

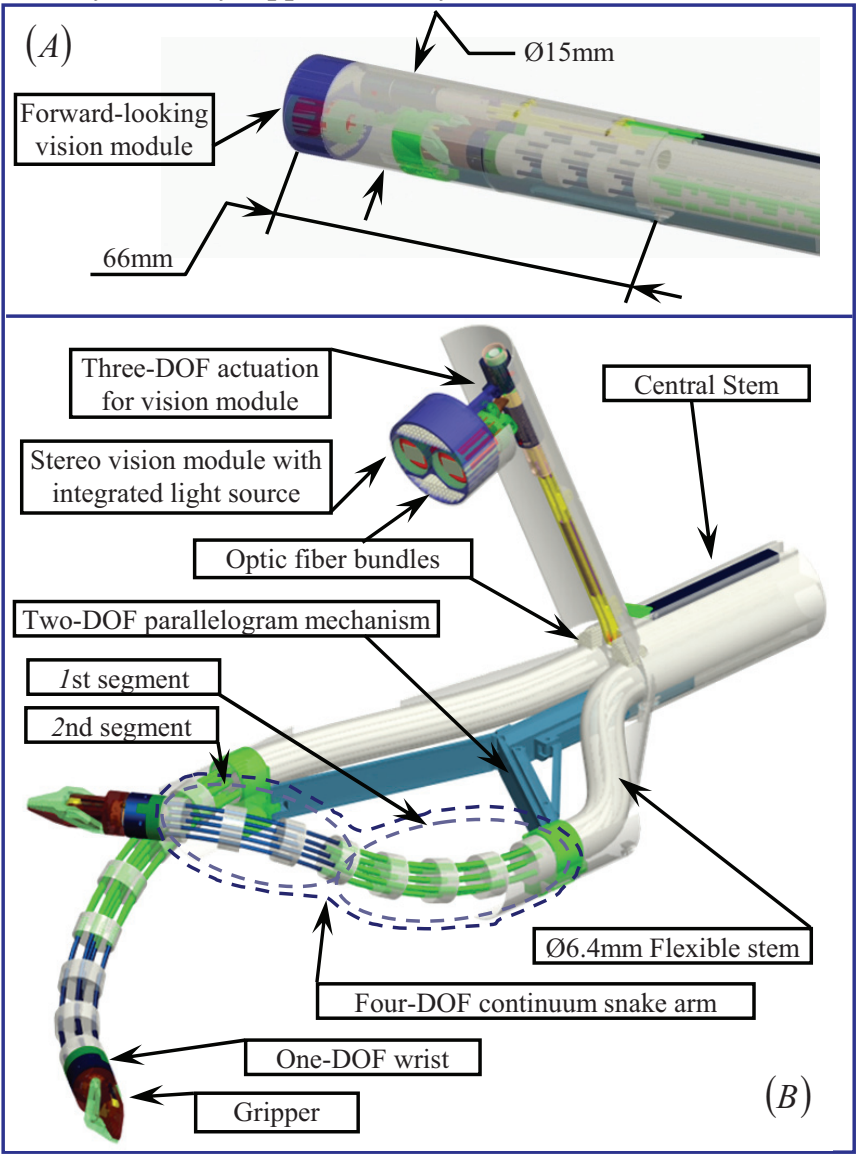

Fig. 1. System Overview of the IREP Robot (A) Folded Configuration (B) Working Configuration

Section II presents the design specifications and system overview of the IREP robot. Design considerations, modification and iterations are detailed in Section III. Section IV presents description, calculation and verifications 
of major system components, preliminary kinematics and workspace analysis. The IREP's control system hierarchy is presented for tele-manipulation in Section $\mathrm{V}$ with conclusions and future works following in Section VI.

\section{DESIGN SpecificAtions AND System OVERVIEW}

Robot-assisted SPA surgery demands the following capabilities: i) the robot should be able to fold for it to pass through a single small skin incision, ii) the robot should be self deployable into a working configuration, iii) target organs and their related tissues (such as gallbladder, hepatic tissues, pancreas, etc.) can be manipulated with enough precision and force, iv) the translational workspace should be bigger than $50 \mathrm{~mm} \times 50 \mathrm{~mm} \times 50 \mathrm{~mm}$ (size of the surgical site on target organs), v) the robot has a stereo vision unit for depth perception and tool tracking, vi) illumination should be integrated as part of the robot design.

The IREP robot of Fig. 1 was conceived to meet these requirements. After a patient's abdomen is insufflated, the IREP robot in its folded configuration can be inserted into the abdomen through a $\varnothing 15 \mathrm{~mm}$ skin incision while using its forward-looking stereo vision module to guide surgeons through the insertion phase. The IREP can unfold itself into a working configuration to perform SPA procedures. An accompanying video (snapshots in I) animates this deployment process. The IREP robot consists of two snakelike continuum robots, two parallelogram mechanisms, and one stereo vision module.

- Each snake-like continuum robot includes four components: i) a gripper, ii) a one-DoF wrist, iii) a fourDoF continuum snake arm and iv) a flexible stem. It acts as a surgical telemanipulation slave for dual arm interventions and delivery of sensors (e.g. ultrasound probe) or energy sources (e.g. cautery). During SPA procedures, each of the arms of the IREP robot can be independently pulled out and replaced with another arm equipped with different surgical end effectors.

- Each parallelogram mechanism has two DoFs for a translational placement of the snake-like continuum robot. The flexible stem will be independently fed in and out to comply with the parallelogram's motion.

- The stereo vision module has a pair of CCD cameras for depth perception as well as tracking of surgical tools. It has three DoFs for pan, tilt, and zoom. A light source using optic fiber bundles is also integrated.

All the controlled joints will be actuated by NiTi tubes or stainless steel rods in push-pull mode. The actuation unit will remain outside patient's body. Motion discrepancy caused by backlash or friction might be compensated as in [20].

\section{Design Considerations AND ITERATIONS}

The IREP robot's final deign was obtained after several rounds of design iterations. The following considerations led to the convergence of the design in Fig. 1.

\section{A. Dimensional Constraints}

The outer diameter of the IREP in folded configuration is $15 \mathrm{~mm}$. This dimension is currently limited by the $\varnothing 6.5 \mathrm{~mm}$ diameter of the CCD cameras (CSH-1.4-V4-END-R1 from NET Inc.) used in the stereo vision module. Placing the cameras axially displaced along the axis of the IREP will make the IREP's insertable portion too long to allow its deployment inside a small cavity. Placing the cameras in parallel will take a diameter of $13 \mathrm{~mm}$ without reserving space for protective covers. Since in [8] a Ø20 $\mathrm{mm}$ incision is available for transumbilical laparoscopic SPA procedures, a diameter of $15 \mathrm{~mm}$ of the IREP is acceptable.

The payload of the four-DoF continuum NiTi snake arms determines the payload of the entire IREP robot since it is the weakest portion of the IREP robot. For this reason, the $\varnothing 6.4 \mathrm{~mm}$ diameter of the four-DoF continuum snake arm was maximized to use all available space in folded configuration. The diameters of the backbones were chosen to be $\varnothing 0.90 \mathrm{~mm}$ for the first segments of the continuum snakes and $\varnothing 0.64 \mathrm{~mm}$ for the distal segments. All backbones are made from super-elastic NiTi tubes to provide channels for actuation of the gripper and the wrist, suction, cautery, light, and delivery of wiring for sensors.

\section{B. Distal Dexterity}

Previous works $[18,20,21]$ demonstrated that continuum snake-like robots as in Fig. 1-(B) can serve as distal dexterity tools for enabling complicated surgical tasks such as suturing and knot tying in confined spaces. The proven dexterity plus the scalability and load-carrying capability of this type of continuum robots as shown in [19] make it an ideal choice for the IREP robot's arms. Furthermore, its intrinsic force sensing capability developed in [22] allows equipping the IREP robot with force sensing capabilities.

\section{Vision Module Placement and Actuation}

To integrate a stereo vision module for tracking surgical tool tip movement, the baseline between the two CCD cameras should be maximized for improved tracking precision. The system configuration with the longest possible baseline is presented in Fig. 2. However, this design has two serious drawbacks. The first drawback is counter intuitive visual perception for the surgeons. This design is not anthropomorphic and providess unintuitive visualization. A more intuitive design imitates the human's anatomy where the eyes visualize an operation site between the arms. The second drawback is that the snake-like continuum robot will obscure a large portion of the field of view and will further complicate the shadows cast by the arms.

Another system configuration was considered as in Fig. 3, where the vision module is lifted upwards to overcome the aforementioned drawbacks. This vision module with a complicated pulley actuation system can provide six DoFs, enabling motions of pan, tilt and zoom for each individual 
camera. Although this six-DoF actuation has advantages such as adjustable baseline; the available space for integrating light source is limited.

The final system configuration is shown Fig. 1, where the two CCD cameras are packed together. A fixed baseline simplifies calibration. Initial simulation showed an accuracy of approximately $0.16 \mathrm{~mm}$ [16]. In addition, a cross sectional area of $36 \mathrm{~mm}^{2}$ in the central stem, now available due to the decrease in actuation lines, can be used for passing optic fiber bundles for illumination.

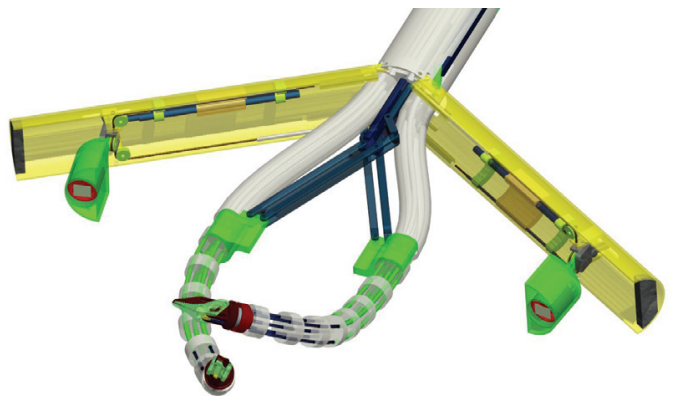

Fig. 2. Abandoned System Configuration \#1

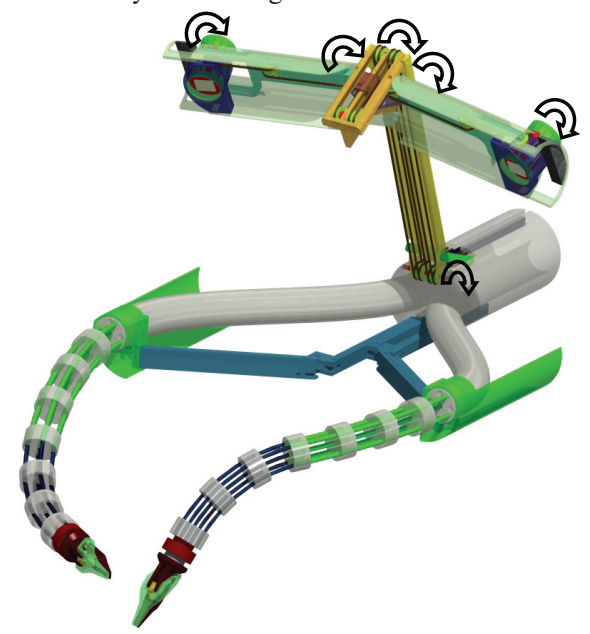

Fig. 3. Abandoned System Configuration \#2

\section{COMPONENT DESCRIPTIONS AND VERIFICATIONS}

This section will present detailed descriptions and the verifications of major components of the IREP robot.

\section{A. Snake-Like Continuum Robot}

The snake-like continuum robot is a key component of the IREP design. It is composed of two segments. Each segment consists of several super-elastic NiTi tubes as backbones and several disks. Fig. 4 shows one segment, where one primary backbone is centrally located and is attached to the base disk and the end disk. Four identical secondary backbones are equidistant from each other and radially spaced from the primary backbone. The secondary backbones are only attached to the end disk and can slide in appropriately toleranced holes in the base disk and in the spacer disks. Two DoF bending motion of this continuum segment is achieved through simultaneous differential actuation of the four secondary backbones. Two segments can be stacked to form the four-DoF continuum snake arm. The backbones of the first and the second segments (shown in Fig. 1) are concentric NiTi super-elastic tubes with outer and inner diameters of $\varnothing 0.90 \times \varnothing 0.76 \mathrm{~mm}$ and $\varnothing 0.64 \times \varnothing 0.51 \mathrm{~mm}$.

In order to determine its design parameters, a workspace analysis will be presented in this subsection to show whether the desired workspace will be covered.

Since the segments are structurally similar, Fig. 4 only shows the $t$ th segment $(t=1,2)$. Nomenclatures are defined in Table I, while coordinate systems are defined as below:

- Base Disk Coordinate System (BDS) of the $t$ th segment $\left\{\hat{\mathbf{x}}_{(t) b}, \hat{\mathbf{y}}_{(t) b}, \hat{\mathbf{z}}_{(t) b}\right\}$ is attached to the base disk of the $t$ th segment, whose XY plane is defined to coincide with the base disk and its origin is at the center of the base disk. The $\hat{\mathbf{x}}_{(t) b}$ points from the center of the base disk to the first secondary backbone while $\hat{\mathbf{z}}_{(t) b}$ is perpendicular to the base disk. The four secondary backbones are numbered according to the definition of $\delta_{(t) i}$.

- Bending Plane Coordinate System (BPS) of the $t$ th segment $\left\{\hat{\mathbf{x}}_{(t) 1}, \hat{\mathbf{y}}_{(t) 1}, \hat{\mathbf{z}}_{(t) 1}\right\}$ is defined such that the continuum segment bends in the $\mathrm{XZ}$ plane, with its origin coinciding with the origin of $\left\{\hat{\mathbf{x}}_{(t) b}, \hat{\mathbf{y}}_{(t) b}, \hat{\mathbf{z}}_{(t) b}\right\}$.

- $\quad$ End Disk Coordinate System (EDS) of the $t$ th segment $\left\{\hat{\mathbf{x}}_{(t) e}, \hat{\mathbf{y}}_{(t) e}, \hat{\mathbf{z}}_{(t) e}\right\}$ is obtained from $\left\{\hat{\mathbf{x}}_{(t) 1}, \hat{\mathbf{y}}_{(t) 1}, \hat{\mathbf{z}}_{(t) 1}\right\}$ by a rotation about $\hat{\mathbf{y}}_{(t) 1}$ such that $\hat{\mathbf{z}}_{(t) 1}$ becomes the backbone tangent at the end disk. The origin of $\left\{\hat{\mathbf{x}}_{(t) e}, \hat{\mathbf{y}}_{(t) e}, \hat{\mathbf{z}}_{(t) e}\right\}$ is at the center of the end disk.

- Gripper Coordinate System (GCS) of the $t$ th segment $\left\{\hat{\mathbf{x}}_{(t) g}, \hat{\mathbf{y}}_{(t) g}, \hat{\mathbf{z}}_{(t) g}\right\}$ is attached to an imaginary gripper affixed to the end disk. $\hat{\mathbf{x}}_{(t) g}$ points from the center of the end disk to the first secondary backbone and $\hat{\mathbf{z}}_{(t) g}$ is normal to the end disk. $\left\{\hat{\mathbf{x}}_{(t) g}, \hat{\mathbf{y}}_{(t) g}, \hat{\mathbf{z}}_{(t) g}\right\}$ is obtained from $\left\{\hat{\mathbf{x}}_{(t) e}, \hat{\mathbf{y}}_{(t) e}, \hat{\mathbf{z}}_{(t) e}\right\}$ by a rotation about $\hat{\mathbf{z}}_{(t) e}$.

We also note that $\left\{\hat{\mathbf{x}}_{(t) g}, \hat{\mathbf{y}}_{(t) g}, \hat{\mathbf{z}}_{(t) g}\right\}$ is identical to $\left\{\hat{\mathbf{x}}_{(t+1) b}\right.$, $\left.\hat{\mathbf{y}}_{(t+1) b}, \hat{\mathbf{z}}_{(t+1) b}\right\}$ when the $t$ th and $(t+1)$ th segment are stacked.

TABLE I

NOMENCLATURE USED IN THIS PAPER

\begin{tabular}{|c|c|}
\hline$i$ & Index of the secondary backbones, $i=1,2,3,4$ \\
\hline$t$ & Index of the segments, $t=1,2, \ldots, n . n=2$ in Fig. 1. \\
\hline$r$ & $\begin{array}{l}\text { Radius of the pitch circle defining the positions of the } \\
\text { secondary backbones in all the disks. }\end{array}$ \\
\hline$\beta$ & $\begin{array}{l}\text { Division angle of the secondary backbones along the } \\
\text { circumference of the pitch circle, } \beta=\pi / 2 \text {. }\end{array}$ \\
\hline$L_{(t)}, L_{(t) i}$ & $\begin{array}{l}\text { Length of the primary and the } i \text { th secondary backbone for } \\
\text { the } t \text { th segment }\end{array}$ \\
\hline $\mathbf{q}_{(t)}$ & $\begin{array}{l}\mathbf{q}_{(t)}=\left[\begin{array}{llll}q_{(t) 1} & q_{(t) 2} & q_{(t) 3} & q_{(t) 4}\end{array}\right]^{T} \text { is the actuation } \\
\text { length vector in the joint space for the } t \text { th segment, where } \\
q_{(t) i} \equiv L_{(t) i}-L_{(t)} .\end{array}$ \\
\hline$\theta_{(t)}(s)$ & $\begin{array}{l}\text { The angle of the tangent to the primary backbone in the } \\
\text { bending plane for the } t \text { th segment. } \theta\left(L_{(t)}\right) \text { and } \theta(0) \text { are }\end{array}$ \\
\hline
\end{tabular}




\begin{tabular}{|c|c|}
\hline & designated by $\theta_{(t) L}$ and $\theta_{0} \cdot \theta_{0}=\pi / 2$ is a constant. \\
\hline$\delta_{(t) i}$ & $\begin{array}{l}\text { For the tth segment, a right-handed rotation angle from } \\
\hat{\mathbf{x}}_{(t) 1} \text { about } \hat{\mathbf{z}}_{(t) 1} \text { to a line passing through the primary } \\
\text { backbone and the } i \text { th secondary backbone. At a straight } \\
\text { configuration } \hat{\mathbf{x}}_{(t) 1} \text { is along the same direction as the } \\
\text { desired instantaneous linear velocity of the end disk. }\end{array}$ \\
\hline$\delta_{(t)}$ & $\delta_{(t)} \equiv \delta_{(t) 1}$ and $\delta_{(t) i}=\delta_{(t)}+(i-1) \beta, i=1,2,3,4$ \\
\hline $\boldsymbol{\Psi}_{(t)}$ & $\begin{array}{l}\boldsymbol{\Psi}_{(t)}=\left[\begin{array}{ll}\theta_{(t) L} & \delta_{(t)}\end{array}\right]^{T} \text { is a configuration vector to define } \\
\text { the pose of the } t \text { th segment of the robot. }\end{array}$ \\
\hline${ }^{1} \mathbf{R}_{2}$ & Rotation matrix of frame 2 with respect to frame 1. \\
\hline${ }^{(t) b} \mathbf{p}_{(t)}(s)$ & $\begin{array}{l}\text { Position vector of a point along the primary backbone in } \\
\text { the } t \text { th segment in }\left\{\hat{\mathbf{x}}_{(t) b}, \hat{\mathbf{y}}_{(t) b}, \hat{\mathbf{z}}_{(t) b}\right\} \cdot{ }^{(t) b} \mathbf{p}_{(t)}\left(L_{(t)}\right) \text { is the } \\
\text { tip position designated by }{ }^{(t) b} \mathbf{p}_{(t) L} .\end{array}$ \\
\hline
\end{tabular}

Thorough analysis of one segment's kinematics can be found in [20, 22]. Only related entities are summarized here.

Shape of the $t$ th continuum segment can be characterized by $\theta_{(t) L}$ and $\delta_{(t)}$ as defined in Table I.

The direct kinematics is provided by:

$$
L_{(t) i}=L_{(t)}+q_{(t) i}=L_{(t)}+r \cos \left(\delta_{(t) i}\right)\left(\theta_{(t) L}-\theta_{0}\right)
$$

Previous works [22] experimentally proved that the continuum segment will bend into a circular shape. The position of the end disk in $\left\{\hat{\mathbf{x}}_{(t) b}, \hat{\mathbf{y}}_{(t) b}, \hat{\mathbf{z}}_{(t) b}\right\}$ can then be derived as:

$$
{ }^{(t) b} \mathbf{p}_{(t) L}=\frac{L_{(t)}}{\theta_{(t) L}-\theta_{0}}\left[\begin{array}{c}
\cos \left(\delta_{(t)}\right)\left(\sin \left(\theta_{(t) L}\right)-1\right) \\
-\sin \left(\delta_{(t)}\right)\left(\sin \left(\theta_{(t) L}\right)-1\right) \\
-\cos \left(\theta_{(t) L}\right)
\end{array}\right]
$$

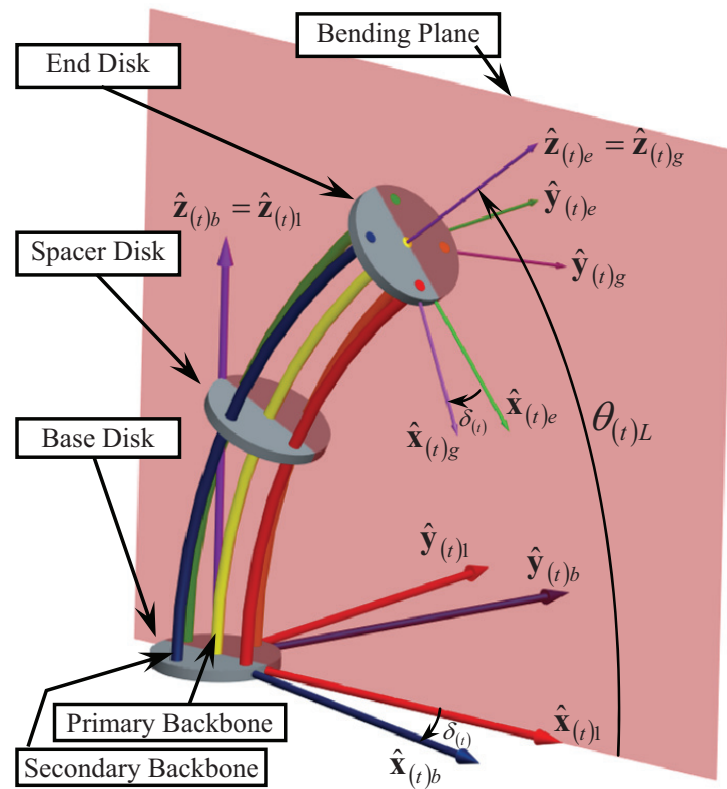

Fig. 4. Nomenclature and Coordinates of the $t$ th Continuum Segment

The rotation matrix ${ }^{(t) b} \mathbf{R}_{(t) g}$ which associates different coordinate systems can be written as the following:

${ }^{(t) b} \mathbf{R}_{(t) g}=\operatorname{Rot}\left(\hat{\mathbf{z}}_{(t) b},-\delta_{(t)}\right) \operatorname{Rot}\left(\hat{\mathbf{y}}_{(t) 1}, \theta_{0}-\theta_{(t) L}\right) \operatorname{Rot}\left(\hat{\mathbf{z}}_{(t) e}, \delta_{(t)}\right)$

Since the wrist and the gripper of the snake-like continuum robot are stacked on top of the 2nd segment's end disk, as shown in Fig. 1, the tip position of the gripper is derived as below, in $\left\{\hat{\mathbf{x}}_{(1) b}, \hat{\mathbf{y}}_{(1) b}, \hat{\mathbf{z}}_{(1) b}\right\}$ :

$$
{ }^{(1) b} \mathbf{p}_{\text {gripper }}={ }^{(1) b} \mathbf{p}_{(1) L}+{ }^{(1) b} \mathbf{R}_{(1) g}\left({ }^{(2) b} \mathbf{p}_{(2) L}+{ }^{(2) b} \mathbf{R}_{(2) g} L_{\text {gw }} \hat{\mathbf{z}}_{(2) g}\right)
$$

Where $L_{g w} \hat{\mathbf{z}}_{(2) g}$ is the distance from the gripper's tip to the origin of $\left\{\hat{\mathbf{x}}_{(2) g}, \hat{\mathbf{y}}_{(2) g}, \hat{\mathbf{z}}_{(2) g}\right\}$.

In the final deign of the snake-like continuum robot, $L_{g w}=15 \mathrm{~mm}, L_{(1)}=35 \mathrm{~mm}$ and $L_{(2)}=25 \mathrm{~mm}$. Length of each segment is determined by the elastic strain limit of the NiTi material under the maximal bending of each segment.

Fig. 5 plots its translational workspace in $\left\{\hat{\mathbf{x}}_{(1) b}, \hat{\mathbf{y}}_{(1) b}, \hat{\mathbf{z}}_{(1) b}\right\}$ generated by scanning the configuration space of $\left\{\theta_{(1) L}, \delta_{(1)}, \theta_{(2) L}, \delta_{(2)}\right\}$. It is an ellipsoidal volume with a somewhat ellipsoidal cavity. Since the two snake-like continuum robots are deployed through the IREP's Ø15mm central stem, their direct implementation will not provide enough overlapped translational workspace. For this reason, two parallelogram mechanisms are included to control the position of the bases of the snake-like continuum robots.

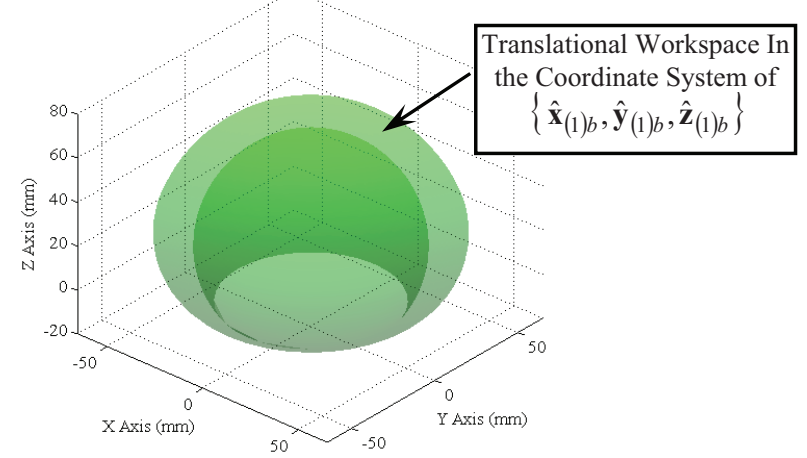

Fig. 5. Translational workspace of the single four-DoF continuum snakelike robot used in the arms of the IREP in Fig. 1

\section{B. Parallelogram Mechanism Design}

The base coordinate $\left\{\hat{\mathbf{x}}_{w}, \hat{\mathbf{y}}_{w}, \hat{\mathbf{z}}_{w}\right\}$ of the IREP robot is attached to the central stem, as shown in Fig. 6. $\hat{\mathbf{z}}_{w}$ is aligned with the stem's axis, while the XY plane is aligned with the stem's cross section at the distal end.

With the actuated parallelogram mechanism, the snakelike continuum robot now can be translated in $\left\{\hat{\mathbf{x}}_{w}, \hat{\mathbf{y}}_{w}, \hat{\mathbf{z}}_{w}\right\}$. Namely, $\quad\left\{\hat{\mathbf{x}}_{(1) b}, \hat{\mathbf{y}}_{(1) b}, \hat{\mathbf{z}}_{(1) b}\right\} \quad$ is obtained by translating $\left\{\hat{\mathbf{x}}_{w}, \hat{\mathbf{y}}_{w}, \hat{\mathbf{z}}_{w}\right\}$ along the following vector:

$$
{ }^{w} \mathbf{p}_{(1) b}=\mathbf{d}_{\text {offset }}+\mathbf{p}_{p r_{-} 1}+\mathbf{R}_{p r_{-} 1} \mathbf{p}_{p r_{-} 2}+\mathbf{h}_{\text {offset }}
$$

Where $\mathbf{d}_{\text {offset }}=\left[\begin{array}{lll}3.8 m m & 0 & 0\end{array}\right]^{T}$ for the left arm; $\mathbf{p}_{p r_{-} 1}=[0$ $\left.\begin{array}{ll}0 & L_{p r}\end{array}\right]^{T}$ provides vertical translation and $L_{p r} \in[5 \mathrm{~mm}$, $30 m m] ; \quad \mathbf{R}_{p r_{-} 1}=\operatorname{Rot}\left(\hat{\mathbf{y}}_{w}, \gamma\right)$ and $\gamma \in\left[0^{\circ}, 60^{\circ}\right]$ for the left $\operatorname{arm} ; \mathbf{p}_{p r_{-} 2}=\left[\begin{array}{lll}0 & 0 & 35 \mathrm{~mm}\end{array}\right]^{T}$ and $\mathbf{h}_{\text {offset }}=\left[\begin{array}{lll}0 & 0 & 5.3 \mathrm{~mm}\end{array}\right]^{T}$.

Combining serially the workspace of the snake-like continuum robot and that of the parallelogram mechanism, the translational workspace of the dual-arm IREP robot is plotted in 0 . The figure shows that the final design fulfills 
the workspace requirement. When the parallelogram mechanism is actuated, the flexible stem will be fed through the central stem by the external actuation system. Key design parameters of the parallelogram, such as $\mathbf{p}_{p r_{-} 2}$ and $L_{p r}$, are determined to be the smallest values which generate the desired workspace.

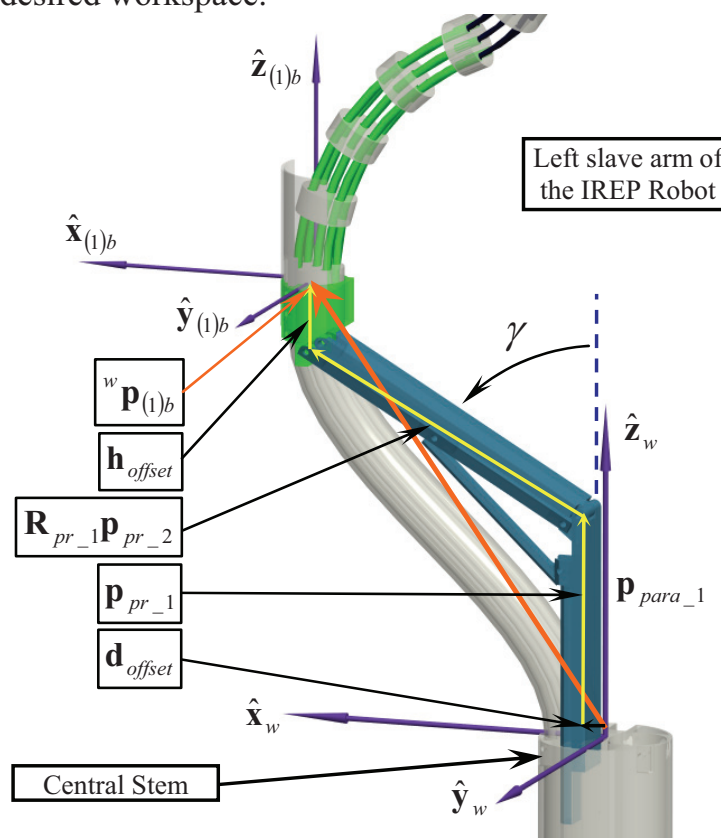

Fig. 6. Diagram of Parallelogram Actuation Calculation

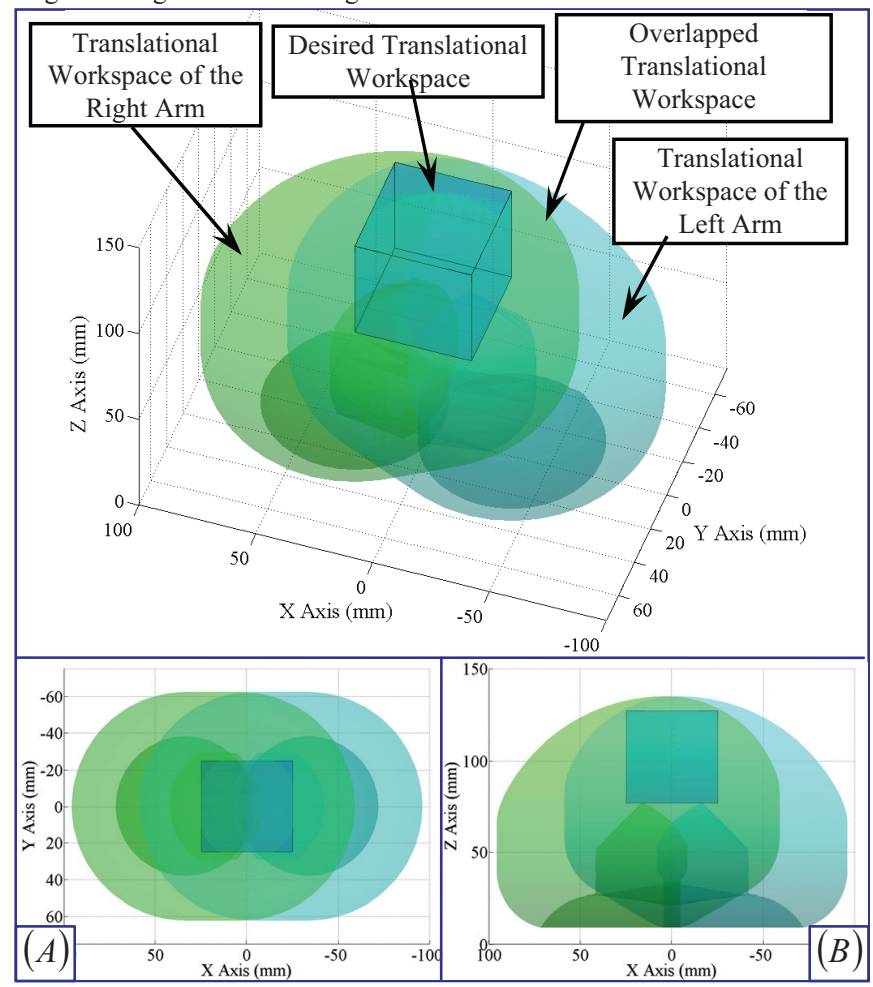

Fig. 7. Translational Workspace of the Dual-Arm IREP Robot: (A) Top View (B) Side View

\section{Gripper Design}

To stabilize a suture, the gripper is expected to provide around $40 \mathrm{~N}$ gripping force [23]. The gripper design then has two requirements: i) the gripper should guarantee $40 \mathrm{~N}$ gripping force with minimal actuation force; ii) it should open as wide as possible. In the final design, a novel solution is presented by creating a slot with two slopes (Fig. 8). When the gripper is actuated by pushing or pulling a NiTi wire, the mechanical advantage of the steep section generates a large gripping force by a small actuation force, while the mild slope portion opens the gripper wide over a short actuation length. This provides a gripper that has wide opening angle and a very large gripping force in a closed configuration.

Simulation was conducted in Pro/E to validate the design. The results are plotted in 0 . From the results, when a gripping force of $40 \mathrm{~N}$ was maintained, the actuation force rapidly declined to $10 \mathrm{~N}$, which can be provided by a $\varnothing 0.4 \mathrm{~mm}$ NiTi Wire. Since this gripper design can only provide enough gripping force when the jaws are almost closed, the teeth heights were designed to accommodate different suturing needle sizes. The gripper's teeth were aligned asymmetrically to ensure three-point contact to stabilize needles with triangular and round cross sections.

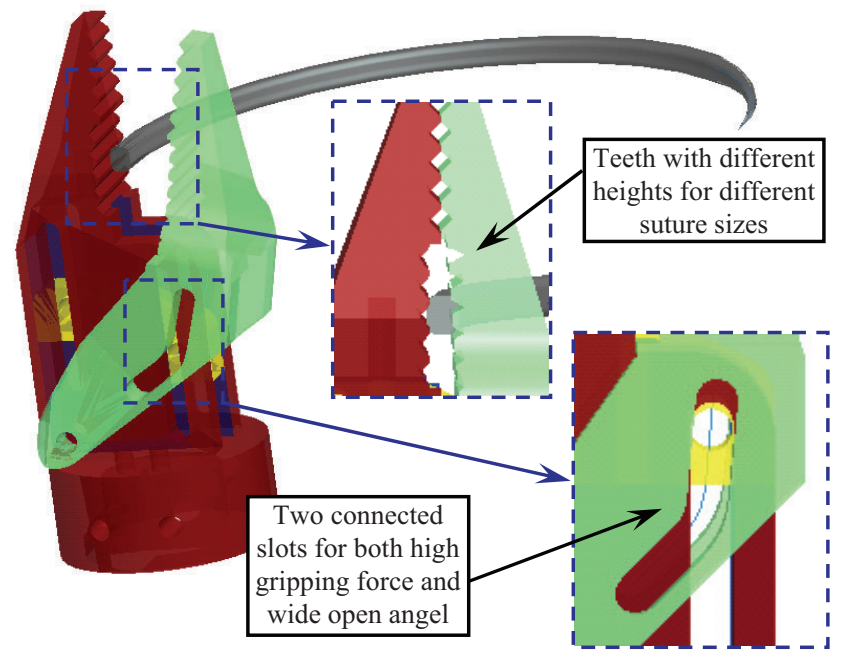

Fig. 8. Gripper Design

\section{Wrist Design:}

A critical component to the dexterity of the IREP robot for fine manipulation tasks (including blunt dissection, dual arm manipulation and suturing) is freedom to rotate an attached surgical end effector, such as the presented gripper, about its longitudinal axis. Previous works [20] showed that the 4-DoF continuum snake arm can transmit axial rotation provided that exact synchronous actuation of all secondary backbones is ensured by proper compensation for model imperfections. However when the parallelogram mechanism opens and deforms the flexible stem, interaction forces will severely affect the torque transmission and the $50 \mathrm{mNm}$ required [24] for suturing may be unavailable. To simplify the design and control of the IREP arms, an independent single DoF wrist located at the distal end of each IREP arm was chosen to meet the functional requirements, including dexterity, actuation speed and payload ability. 
This wrist design presents a unique challenge for robotic mechanisms of this size. Critical factors constraining the wrist design included payload, a maximum overall outside diameter defined by the external superstructure and a requirement for robustness and smooth operation in the surgical environment. Multiple designs were considered including a geared mechanism given in I.A. The limitations of providing torsion through the continuum robot, while maintaining stiffness at the wrist and joint velocity per clinical needs proved infeasible.

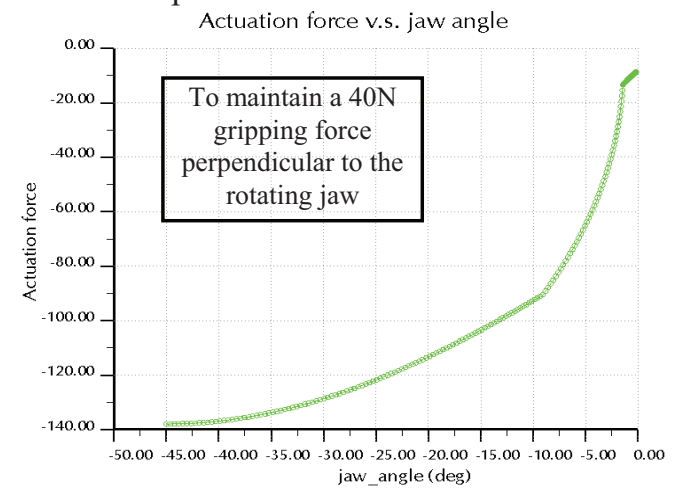

Fig. 9. Gripper Actuation Force while Maintaining a 40N Gripping Force

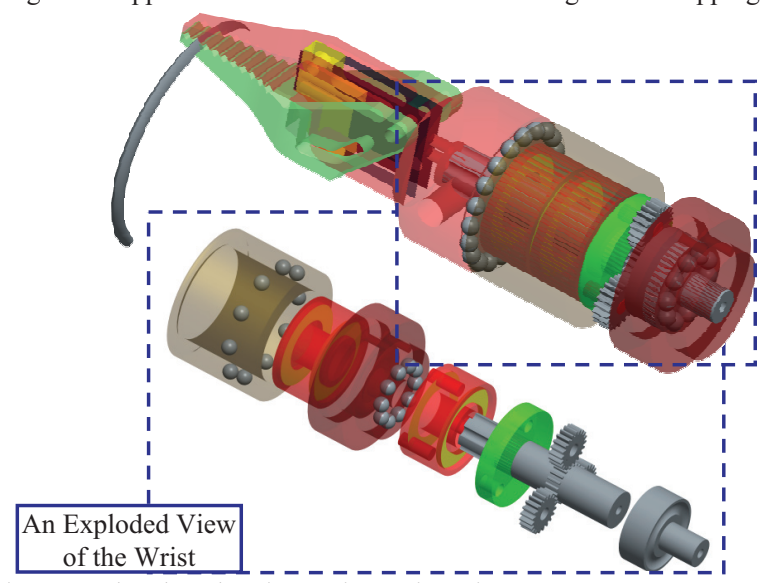

Fig. 10. Abandoned Wrist Design Using Tiny Gears

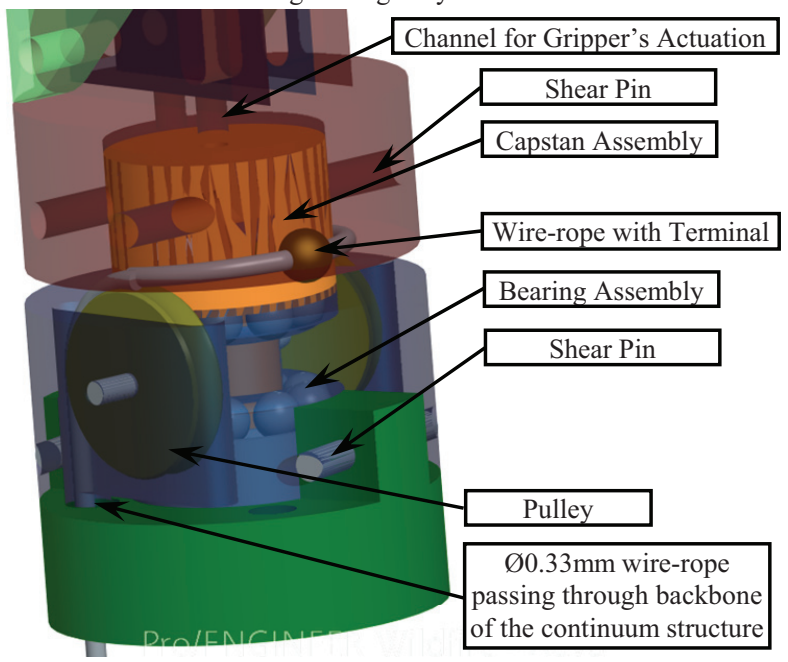

Fig. 11. Final Wrist Design Indicating Major Components

The design converged to a capstan design shown in I.A. The proposed design achieves axial rotation and delivers torque via a $\varnothing 0.33 \mathrm{~mm}$ steel wire-rope running over pulleys and around a capstan arranged axially in line with the gripper. This design achieves approximately $150^{\circ}$ of axial rotation, suitable for manipulation in SPA surgery.

\section{E. Deployment Simulation}

The IREP robot can be inserted into patient's abdominal cavity in its folded configuration and then be unfolded into its working configuration. The accompanying movie clip animates the deployment process. I presents snapshots from the accompanying animation.

\section{CONTROL SySTEM HIERARCHY}

The control system of the IREP robot uses a real time host-target environment powered by $\mathrm{xPC}$ Target ${ }^{\mathrm{TM}}$ from The MathWorks, Inc. The planned control hierarchy is presented in Fig. 13. A GUI running on the host PC takes motion inputs from two master manipulators and then sends them to the target PC via ethernet connection after scaling and mapping. Target PC processes the desired motions $\mathbf{x}_{d}$ of the IREP robot by solving kinematics and redundancy resolution in a $1 \mathrm{kHz}$ servo control loop. A third PC running vision processing module will output the stereo display and feed tool tracking results $\mathbf{x}_{v}$ to the host PC for future motion compensation of the IREP's dual snake-like arm.

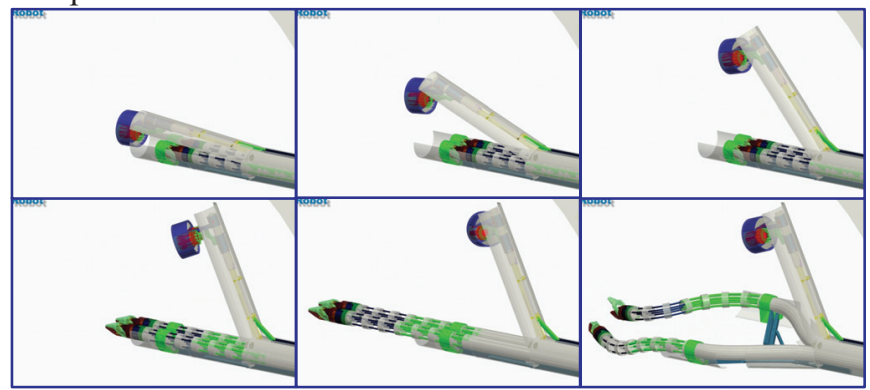

Fig. 12. An image sequence showing the deployment of the IREP Robot as viewed in the accompanying video

\section{CONCLUSION AND FUTURE WORKS}

Single Port Access (SPA) surgery requires new technology that overcomes space, visualization, and dexterity constraints. This paper presented a novel design, workspace analysis and deployment animation of a 17-DoF Insertable Robotic Effector Platform (IREP) for SPA Surgeries. Design considerations and iterations, major component description and verification, as well as control system hierarchy were presented. The proposed design has already taken safety issues into considerations. For example, the 5-backbone design of the snake-like continuum robot can continue to function even after one NiTi backbone is broken. Moreover, once disconnected from the malfunctioned actuation unit, all the joints of the IREP robot can be manually actuated to reverse the deployment sequence. The IREP robot can then be pulled out of the patient's abdomen in a safe, enclosed configuration.

Initial simulation results verified that the design meets all design specifications. Comprehensive simulations including 
dexterity evaluation, suturing verification, actuation redundancy resolution will be carried out in the near future.

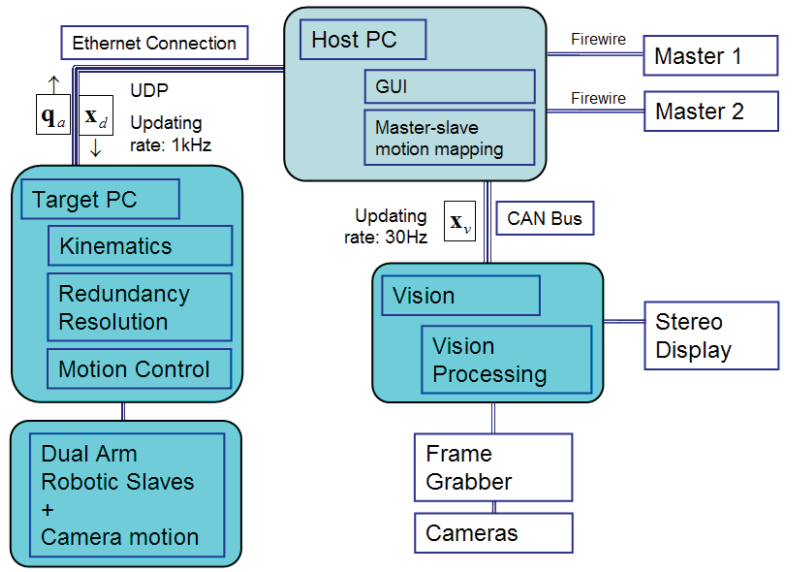

Fig. 13. Control System Hierarchy for the IREP Robot

\section{ACKNOWLEDGMENT}

The authors appreciate Nancy J. Hogle's help in project development and lab support.

\section{REFERENCES}

[1] D. J. Deziel, K. W. Millikan, S. G. Economou, M. A. Doolas, S.-T. Ko, and M. C. Airan, "Complications of Laparoscopic Cholecystectomy: A National Survey of 4,292 Hospitals and an Analysis of 77,604 Cases," The American Journal of Surgery, vol. 165, pp. 9-14, Jan 1993.

[2] M. J. Mack, "Minimally Invasive and Robotic Surgery," The Journal of the American Medical Association, vol. 285, pp. 568-572, Feb 72001.

[3] E. Berber, K. L. Engle, A. Garland, A. String, A. Foroutani, J. M. Pearl, and A. E. Siperstein, "A Critical Analysis of Intraoperative Time Utilization in Laparoscopic Cholecystectomy," Surgical Endoscopy, vol. 15, pp. 161-165, 2004.

[4] M. Bessler, P. D. Stevens, L. Milone, M. Parikh, and D. L. Fowler, "Transvaginal Laparoscopically Assisted Endoscopic Cholecystectomy: a Hybrid Approach to Natural Orifice Surgery," Gastrointestinal Endoscopy, vol. 66, pp. 1243-1245, Dec 2007.

[5] D. J. Abbott, C. Becke, R. I. Rothstein, and W. J. Peine, "Design of an Endoluminal NOTES Robotic System," in IEEE/RSJ International Conference on Intelligent Robots and Systems (IROS), San Diego, CA, USA, 2007, pp. 410 - 416.

[6] S. C. Low, S. W. Tang, Z. M. Thant, L. Pjhee, K. Y. Ho, and S. C. Chung, "Master Slave Robotic System for Theraputic nGastrointestinal Endoscopic Procedures," in 28th IEEE/EMBS Annual International Conference New York City, NY, 2006.

[7] G. Navarra, E. Pozza, S. Occhionorelli, P. Carcoforo, and I. Donini, "One-Wound Laparoscopic Cholecystectomy," British Journal of Surgery, vol. 84, p. 695, 1997.

[8] A. A. Gumbs, L. Milone, P. Sinha, and M. Bessler, "Totally Transumbilical Laparoscopic Cholecystectomy," Journal of Gastrointestinal Surgery, Aug 2008.

[9] R. H. Taylor and D. Stoianovici, "Medical Robotics in Computer-Integrated Surgery," IEEE Transactions on Robotics and Automation, vol. 19, pp. 765-781, 2003.

[10] G. S. Guthart and K. J. Salisbury, "The Intuitive ${ }^{\mathrm{TM}}$ Telesurgery System: Overview and Application," in IEEE
International Conference on Robotics and Automation (ICRA), San Francisco, CA, 2000, pp. 618-621.

[11] G. Hubens, H. Coveliers, L. Balliu, M. Ruppert, and W. Vaneerdeweg, "A Performance Study Comparing Manual and Robotically Assisted Laparoscopic Surgery Using the da Vinci System," Surgical Endoscopy, vol. 17, pp. 1595-1599, Oct 2003.

[12] M. Saad, "Fisherman's Technique, Introducing a Novel Method for Using the Umbilical Port for Removal of Appendix During Laparoscopic Appendectomy," Surgical Laparoscopy Endoscopy \& Percutaneous Techniques, vol. 17, pp. 422-424, Oct 2007.

[13] F. C. Becerra Garcia, M. C. Misra, H. K. Bhattacharjee, and G. Buess, "Experimental Trial of Transvaginal Cholecystectomy: an Ex Vivo Analysis of the Learning Process for a Novel Single-Port Technique," Surgical Endoscopy, vol. Ahead of Print, Jan 2009.

[14] T. Hu, P. K. Allen, and D. L. Fowler, "In-Vivo Pan/Tilt Endoscope with Integrated Light Source," in IEEE/RSJ International Conference on Intelligent Robots and Systems (IROS), San Diego, CA, USA, 2007, pp. 1284-1289.

[15] N. J. Hogle, T. Hu, P. K. Allen, and D. L. Fowler, "Comparison of Monoscopic Insertable, Remotely Controlled Imaging Device with a Standard Laparoscope in a Porcine Model," Surgical Innovation, vol. 15, pp. 271-276, Dec 2008.

[16] T. Hu, P. K. Allen, N. J. Hogle, and D. L. Fowler, "Insertable Surgical Imaging Device with Pan, Tilt, Zoom, and Lighting," in IEEE International Conference on Robotics and Automation (ICRA), Pasadena, CA, USA, 2008, pp. 2948-2953.

[17] T. Hu, P. K. Allen, T. Nadkarni, N. J. Hogle, and D. L. Fowler, "Insertable Stereoscopic 3D Surgical Imaging Device with Pan and Tilt," in IEEE / RAS-EMBS International Conference on Biomedical Robotics and Biomechatronics (BIOROB), Scottsdale, AZ, USA, 2008, pp. 311-316.

[18] A. Kapoor, N. Simaan, and R. H. Taylor, "Suturing in Confined Spaces: Constrained Motion Control of a Hybrid 8DoF Robot," in International Conference on Advanced Robotics (IACR), Seattle, USA, 2005, pp. 452-459.

[19] N. Simaan, "Snake-Like Units Using Flexible Backbones and Actuation Redundancy for Enhanced Miniaturization," in IEEE International Conference on Robotics and Automation (ICRA), Barcelona, Spain, 2005, pp. 3012-3017.

[20] N. Simaan, K. Xu, A. Kapoor, W. Wei, P. Kazanzides, P. Flint, and R. H. Taylor, "Design and Integration of a Telerobotic System for Minimally Invasive Surgery of the Throat " International Journal of Robotics Research, vol. Accepted for Publication. First published online on May 27th through IJRR OnlineFirst, 2009.

[21] K. Xu and N. Simaan, "Actuation Compensation for Flexible Surgical Snake-like Robots with Redundant Remote Actuation," in IEEE International Conference on Robotics and Automation (ICRA), Orlando, Florida, USA, 2006, pp. 41484154.

[22] K. Xu and N. Simaan, "An Investigation of the Intrinsic Force Sensing Capabilities of Continuum Robots," IEEE Transactions on Robotics, vol. 24, pp. 576-587, June 2008.

[23] D. Sallé, F. Cepolina, and P. Bidaud, "Surgery Grippers for Minimally Invasive Heart Surgery," in IEEE International Conference on Intelligent Manipulation and Grasping (IMG), Genova, Italy, 2004.

[24] M. Tavakoli, R. V. Patel, and M. Moallem, "Robotic Suturing Forces in the Presence of Haptic Feedback and Sensory Substitution," in IEEE Conference on Control Applications, Toronto, Canada, 2005, pp. 1-6. 